\title{
Verzeichnis der Absender
}

unter Angabe der Briefnummer

Appelius, Johann Wilhelm 150

Arnoldt, Daniel Heinrich 169

Behr, Georg Heinrich 156

Berger, Christian Gotthelf 93

Beyer, Justus Israel 25, 48

Biedermann, Johann Gottlieb 5, 99, 153, 172

Bodmer, Johann Jakob 18, 81, 162, 176

Breitinger, Johann Jakob 184

Brucker, Jakob 13, 16, 38, 80, 86, 88, 109, 118, 133, 166

Burgmann, Joachim Heinrich 84

Clodius, Christian 29

Cocceji, Samuel von 63

Denso, Johann Daniel 20

Deutsche Gesellschaft in Leipzig 71, 75

Drollinger, Karl Friedrich 147

Duncan, Johann Christoph 110

Eberlein, Christian Ludwig 11

Elsner, Jacob 36, 52

Fabricius, Johann 32

Falckenhagen, Adam 102, 154

Flottwell, Cölestin Christian 35, 123, 163

Gottschald, Johann Jakob 139

Gottsched, Johann Christoph

- an die Berliner Sozietät der Wissenschaften 108

- an die Deutsche Gesellschaft in Leipzig 65, 73

- an Bodmer, Johann Jakob 54, 174

- an Manteuffel, Ernst Christoph von $8,21,27,41,58,72,112,119,130$, 136, 157, 168, 179, 185, 194, 198

- an May, Johann Friedrich 82

Gottsched, Luise Adelgunde Victorie

- an Manteuffel, Ernst Christoph von $113,131,140,146,158,180,183$, 189, 196

Hassen, Martin 124

Hoffmann, Balthasar 103

Holtzendorff, Christian Gottlieb von 44, 138

Hudemann, Ludwig Friedrich 137

Janus, Christian Friedrich Jakob 42, 57, 101,202

de Jariges, Philippe Joseph 121

Jauna, Dominique 17

Jerusalem, Johann Friedrich Wilhelm 23

John, Johann Siegmund 10

Kahle, Ludwig Martin 12

Kemna, Ludolf Bernhard 104, 111, 135

Kopp, Johann Friedrich 69, 177, 203

Krohse, Johann Andreas 201

Kulmus, Johann Adam 105

Kulmus, Johann Ernst 106

Lamprecht, Jacob Friedrich 53

Lange, Karl Heinrich 39, 127

Lemker, Heinrich Christian 94

Lindner, Kaspar Gottlieb 64

Ludovici, Karl Günther 2

Maichel, Daniel 7, 33, 92, 160

Manhardt, Johann Kaspar 49

Manteuffel, Ernst Christoph von 1, 6, 9, 26, 28, 55, 66, 76, 107, 114, 116, $122,126,132,134,142,143,145$, 151, 161, 182, 186, 187, 193, 197, 200, 204

Marschall (Marschalch), Heinrich Wilhelm von 173 
Meiern, Johann Gottfried von 22

Mencke, Friedrich Otto 164

Metschel, Johann Christian 100

Metzler, Daniel Gottlieb 181

Minor, Melchior Gottlieb 188

Möhring, Paul Heinrich Gerhard 159

Mosheim, Johann Lorenz 45, 77, 85, 97

Neuber, Johann 167

Pfaff, Christoph Matthäus 148

Poley, Heinrich Engelhard 60, 128

Pyra, Immanuel Jacob 83

Reinbeck, Johann Gustav 152

Richey, Michael 96

Rohr, Julius Bernhard von 24

Rolief, Johann Wilhelm 46

Rosenberg, Albrecht von 70, 87

Scharff, Gottfried Balthasar 40, 61, 78

Scheibe, Johann Adolph 190

Schindel, Johann Christian 34, 149, 178

Schmidt, Johann Christoph 199

Schneider, Johann Kaspar 56
Schwabe, Johann Joachim 3, 4, 14, 15 , 50

Schwäneschuch, Christian Franz 175

Schwartz, Albrecht Georg 165

Schwarz, Johann Christoph 115, 120

Seelen, Johann Heinrich von 51

Siemsen, Joachim Heinrich 144

Stein, Johann Friedrich 117

Steinauer, Johann Wilhelm 79, 91, 195

Stoppe, Daniel 37, 74

Strimesius, Johann Samuel 191

Suke, Christoph Gerhard 19

Suke, Lorenz Henning 67, 98, 125,170

Thamm, Christian Bernhard 62

Venzky, Georg 43, 59, 155

Volkelt, Johann Gottfried 30, 47, 68

Weichmann, Christian Friedrich 90, 95

Weichmann, Friedrich 89, 129, 141, 171, 192

Zedtwitz, Christian Friedrich 31 